\title{
ANTIGENO SOLUBLE Mycobacterium leprae Y PPD EN ESCOLARES DE LA ISLA DE PASCUA Y SANTIAGO DE CHILE, $1987^{1}$
}

\author{
HUGO HURTADO G.* , LEONARDO RODRIGUEZ G.**
}

\begin{abstract}
Se midió la reacción cruzada de los antígenos proteina pura derivada de tuberculina (PPD) y antígeno soluble de Mycobacterium leprae (ASML) mediante dos muestras de niños entre 6-14 años: una en un área endémica (Isla de Pascua) y la otra en una no endémica (Santiago de Chile continental). Cada grupo de niños se inoculó simultáneamente con PPD y ASML en los antebrazos derecho e izquierdo respectivamente. Las lecturas fueron hechas $\mathbf{4 8}$ horas después. Los análisis estadísticos mostraron una evidente reacción cruzada de los dos antígenos. Se presentó también una significativa mayor reacción en el área endémica (Pascua) que en Santiago tanto para PPD como para ASML.
\end{abstract}

\section{INTRODUCCION}

Chile es un territorio libre de lepra con la sola excepción de la Isla de Pascua, territorio insular distante $5000 \mathrm{~km}$ de las costas de Chile continental (1).

La endemia leprótica en la Isla de Pascua se inició a finales del siglo pasado con casos provenientes de la Polinesia (4). Tanto en Chile continental como en la Isla de Pascua la tuberculosis presenta una prevalencia significativa.

En el Instituto Nacional de Dermatología de Venezuela, se ha desarrollado una prueba intradérmica con un antígeno soluble de Mycobacterium leprae (ASML) cuya sensibilidad mediada por linfocitos a $M$. leprae es homologable a la prueba intradérmica con PPD para TBC.

Los antecedentes bibliográficos evidencian que la prueba intradérmica con PPD positivo (por sobre 10 mm de induración) tiene una buena correlación con la presencia de mecanismos de defensa inmunocelular al bacilo de Koch (5). De igual forma, la hipótesis de trabajo en el uso de ASML, plantea que existe también una correlación entre su positividad y la presencia de mecanismos de defensa inmunocelular al bacilo de Hansen (2).

Estas pruebas intradérmicas permitirían identificar grupos poblacionales de alto riesgo, en los que se podría concentrar las acciones de prevención y de pesquisa de estas enfermedades. Así ha sido posible un manejo racional y de buena calidad del programa de inmunoprofilaxis para TBC en Chile, y la posibilidad de contar con una herramienta epidemiológica similar para lepra, implicaría un aporte significativo, preliminar al desarrollo de un programa de inmunoprofilaxis en lepra. Estas consideraciones señalan la gran importancia de poder contar con métodos epidemiológicos que permitan evaluar la especificidad de ASML. La situación en que se cuenta con dos grupos poblacionales comparables: uno de una zona endémica de lepra (Isla de Pascua) y otro de una zona no endémica (Chile continental), es peculiar y de gran valor para evaluar esta prueba intradérmica.

Por ello se realizó un ensayo que consideró la aplicación de las pruebas intradérmicas de PPD y de ASMIL en dos grupos de menores (escolares de 6 a 14

1 Esta investigación obtuvo financiación parcial de TDRMHO.

- Profesor Asociado, Departamento de Medicina Social, Facultad de Salud, Universidad del Valle, Cali, Colombia.

** Ex-Director Médico de la Isla de Pascua, Universidad de Chile, Santiago de Chile. 
años) en la Isla de Pascua y Santiago de Chile, de manera que el estudio de las correlaciones de las respuestas a ambos antígenos en ambos grupos, permitieran medir la reacción cruzada que pudiera tener el ASML con respecto a PPD e indirectamente poder plantear los niveles de especificidad que presentaría el ASML.

\section{MATERIAL Y METODOS}

A finales de 1984 se identificó a dos grupos de escolares: uno que correspondía a todos los niños (115) de 6 a 14 años de la única escuela de la Isla de Pascua y otro grupo de 167 niños en el mismo rango de edad de una escuela de Santiago de Chile. La totalidad de los menores de ambos grupos presentaban cicatriz de vacunación BCG; se les aplicó las pruebas intradérmicas con PPD y ASML en la cantidad de $0.1 \mathrm{ml}$ con antígenos obtenidos a través de la OPS.

El PPD se aplicó en el tercio medio de la cara anterior del antebrazo izquierdo y simultáneamente se aplicó el ASML en el tercio medio de la cara anterior del antebrazo derecho. En ambas inoculaciones se usó material diferente con agujas No. 27 y fueron leidas a las 48 horas. La reacción se midió en mm de induración mediante palpación y medición con regla transparente milimétrica; se consideró positivo $10 \mathrm{~mm}$ o más para PPD y 15 o más para ASML.

Los resultados fueron tabulados y analizados en computadora con el programa SAS (Statistical Analisys System) mediante el análisis de correlación, regresión, normalidad y pruebas de medias y correlaciones.

\section{RESULTADOS}

Se presenta el análisis de 115 niños de la Isla Pascua y 149 niños de Santiago de Chile, que tenían completos todos sus datos. En el Cuadro 1 se resumen la mayoría de los análisis estadísticos posibles concernientes a la correlación de ASML con respecto a PPD, es decir, a la reacción cruzada que según el presente estudio parecen tener estos dos antígenos:

1. No existen diferencias significativas para la Isla de Pascua y Santiago de Chile, entre los promedios de edad 9.5, $9.7(\mathrm{p}=0.21)$, lo que hace a estos dos grupos comparables en cuanto a la variable edad.
Cuadro 1

Estadísticas descriptivas según grupo y antígeno

$\mathrm{Ch}^{\mathrm{N}}{ }^{\mathrm{N}} \mathrm{P} \quad \mathrm{Ch}{ }^{\mathrm{X}} \mathrm{P} \quad \mathrm{Ch}{ }^{\mathrm{S}} \mathrm{P} \quad \mathrm{Ch}{ }^{\mathrm{P}}$

$\begin{array}{lrrrrrrrr}\text { ASML } & 153 & 115 & 3.05 & 8.0 & 5.1 & 8.2 & 0.23 & \\ \text { PPD } & 149 & 115 & 7.23 & 10.6 & 5.7 & 9.0 & & 0.4 \\ \text { Edad } & 167 & 115 & 9.7 & 9.5 & 1.9 & 2.5 & \end{array}$

$\mathrm{Ch}=$ Chile

$\mathrm{P}=$ Isla de Pascua

$\mathrm{N}=$ Número de niños

$\mathrm{X}=$ Promedio de induración ( $\mathrm{mm}$ )

$\mathrm{r}^{2}=$ Coeficiente de determinación

$\mathrm{S}=$ Desviación estándar

2. Entre los dos grupos en estudio hay evidencias de diferencias significativas tanto para reacción a ASML como a PPD, con promedio mayor de induración en la Isla de Pascua.

3. La reacción promedio en $\mathrm{mm}$ de induración es significativamente mayor para PPD que para ASML, tanto en Chile como en la Isla de Pascua $(\mathrm{p}=0.001)$.

4. Existe un coeficiente de correlación (r) significativo de 0.48 entre ASML y PPD en Chile ( $\mathrm{p}=$ 0.001 ). Su coeficiente de determinación $\mathrm{r}^{2}=0.23$.

5. También para la Isla de Pascua se presenta un coeficiente de correlación de Pearson (r) significativo de 0.63 entre los dos antígenos $(\mathrm{p}=0.001)$. Su coeficiente de determinación $\mathrm{r}^{2}=0.40$.

6. Entre los dos coeficientes de correlación ( 0.48 para Santiago y 0.63 para la Isla de Pascua) no hay evidencia de diferencia significativa. Es decir, se presenta la misma fuerza de asociación entre los dos antígenos en Santiago como la Isla de Pascua $(\mathrm{p}=0.08)$.

Para Chile continental, de los 8 niños positivos a ASML (Cuadro 2) 7 de ellos también son positivos a $\mathrm{PPD}$, con rango duración de $12-20 \mathrm{~mm}$. Sólo hay uno sin respuesta a PPD y $21 \mathrm{~mm}$ a ASML. 
En los 149 niños vale la pena destacar que hubo 15 (10.1\%) con respuesta nula a ASML y positivos para PPD. En cuanto a reacciones nulas $90(58.8 \%)$ no reaccionaron a ASML, $28(18.8 \%)$ a PPD y 21 (14.1\%) a ninguno de los dos antígenos. Se demuestra una fuerte asociación entre los dos antígenos para Chile continental, con alta significancia estadística (Cuadro 3).

Para la Isla de Pascua, los porcentajes de positividad fueron de $30 \%$ para ASML, $47 \%$ para PPD y $26.1 \%$ para ambos antígenos (Cuadro 4). De los 35 niños positivos a ASML, 30 también Ios fueron a PPD, con rangos de induración de 10 a $23 \mathrm{~mm}$. De los 5 restantes, negativos a PPD, 2 dieron respuesta nula y los otros 3 niños 5 , 6 y $7 \mathrm{~mm}$ respectivamente. Del total de 115 niños también vale la pena recalcar que hubo $8(7 \%)$ con respuesta nula a ASML y positivos a PPD.

De las reacciones nulas $45(39 \%)$ niños no reaccionaron a ASML, $32(27.8 \%)$ a PPD y 27 (23.5\%) a ninguno de los dos antígenos. También se demuestra una fuerte asociación entre los dos antígenos para la Isla de Pascua, con alta significancia estadística (Cuadro 4). Para ambos grupos, Isla de Pascua y Chile

\section{Cuadro 2}

Ocho niños positivos a ASML según edad y reacción a PPD. Chile

$\begin{array}{lcccccccc}\text { Edad } & 11 & 09 & 10 & 07 & 12 & 10 & 07 & 10 \\ \text { PPD } & 0 & 12 & 14 & 15 & 16 & 17 & 20 & 20 \\ \text { ASML } & 21 & 17 & 15 & 15 & 18 & 24 & 18 & 18\end{array}$

Cuadro 3

Sero-reacción a PPD y ASMI, 149 niños. Chile

\begin{tabular}{|c|c|c|c|c|}
\hline \multicolumn{5}{|c|}{ ASML } \\
\hline & & - & + & Total \\
\hline & - & 103 & 1 & 104 \\
\hline \multicolumn{5}{|l|}{ PPD } \\
\hline & + & 38 & 7 & 45 \\
\hline Total & & 141 & 8 & 149 \\
\hline \multicolumn{5}{|c|}{ Prueba exacta Fisher valor $p=0.0009$} \\
\hline \multicolumn{5}{|c|}{ Mantel-Haenszel $=13$ o8 valor $p=0.00029$} \\
\hline
\end{tabular}

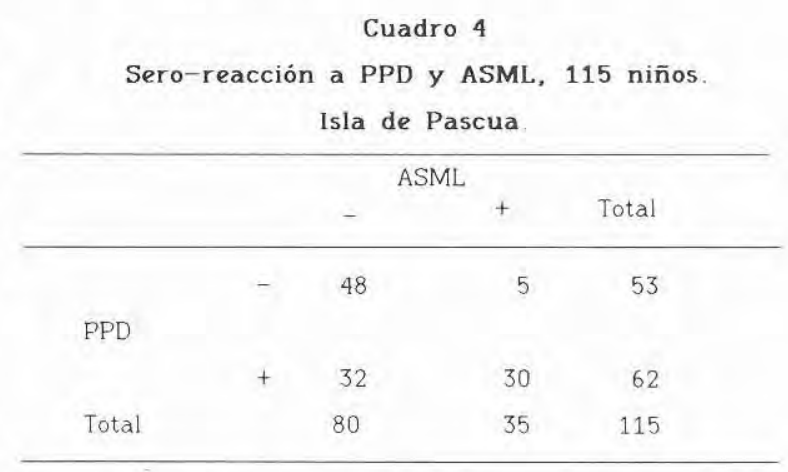

$\dot{x}^{2}=20.478$ valor $p=0.000006$

Mantel-Haenszel $=20.30$ valor $p=0.000006$

continental, se calcularon los coeficientes de correlación entre los negativos a PPD y su respectiva reacción a ASML, dando para la Isla de Pascua 0.38 ( $\mathrm{p}=$ $0.01)$ y para Chile 0.05 y no significativo.

\section{DISCUSION Y ANALISIS}

De acuerdo al análisis de los resultados del presente estudio parece evidente una reacción cruzada entre los dos antígenos. Después de analizarlo en los puntos 4 , 5 y 6 del Cuadro 2 se ve que de los positivos a ASML, en Chile 7 de $8(87.5 \%)$ son positivos a PPD y en la Isla de Pascua 30 de $35(85.7 \%)$ también son positivos a PPD, luego no parece ser que ASML sea específico para detectar agentes inmunológicos medidos por linfocitos a $M$. leprae, cruzándose con los del $M$. tuberculosis. Son muy escasos los casos en que da una reacción pura a ASML: 1 caso en Chile y 2 en la Isla de Pascua.

En Chile, zona no endémica, 23\% de las reacciones a ASML son explicadas por la reacción cruzada a PPD y en la Isla de Pascua, zona endémica, $40 \%$ de acuerdo con los coeficientes de determinación encontrados significativos en el análisis de correlación. Representando, como dice Wrich y col (3): "una desventaja enorme por las dificultades para atribuir estas respuestas no específicas a fuentes apropiadas, y existiendo escasas razones para recomendar el uso de estas preparaciones antígenas de $M$. leprae". No se ha podido demostrar una correlación cuantitativa absoluta entre el diámetro de las reacciones intradérmicas y el grado de resistencia (3).

La ausencia de antígenos específicos a $M$. leprae, en cuanto a evaluación de inmunoterapia e inmunopro- 
filaxis, hace muy difícil saber cuáles micobacterias tienen antígenos necesarios para protección contra $M$. leprae (3).

Según Pattyn (1), los estudios de inmunodifusión no han mostrado ningún antígeno específico a $M$. leprae. Se han encontrado antígenos cruzados por inmunoelectroforesis en reacción cruzada con otras micobacterias, con particular situación para antígeno 21 relacionado a $M$. tuberculosis.

La reacción tanto a PPD como a ASML más alta en la Isla Pascua que en Chile continental, quizá es debido a la gran manipulación antígena en especial para TBC que ha ocurrido en esta zona, como se ve en los diferentes informes del Ministerio de Salud de Chile sobre esta Isla, además de ser una zona endémica (4).

\section{CONCLUSIONES}

Existen escasas razones para recomendar el uso de este antígeno soluble solo, sin el control de PPD. Era de esperarse este cruce porque la literatura señala para las pruebas intradérmicas no muy buena sensibilidad y especificidad (3), además de reacción cruzada para otras especies de micobacterias.

Merecen atención especial las preparaciones químicamente definidas sobre todo si poseen determinantes aparentemente específicos para $M$. leprae. Es necesario la definición de antígenos específicos que permitan medir exactamente la respuesta a anticuerpos específicos en las diversas etapas de infección y enfermedad clínica que permita la aplicación a grandes grupos de población, además de encontrar métodos de transmisión. Las pruebas intradérmicas no son sensibilizantes y no parecen alterar el estado inmunológico de las personas examinadas; el hallar pruebas intradérmicas específicas permitiría evaluar tanto la efectividad como la duración de la inmunoterapia y la inmunoprofilaxis. La especificidad no es clara por lo menos para el presente estudio.

En conclusión, se puede decir que la evaluación epidemiológica de la lepra esta sujeta a muchos problemas, el más importante de los cuales es la ausencia de una herramienta específica para identificar la infección subclínica y las dificultades para definir exactamente los diversos tipos y estadíos de la enfermedad, especialmente en los puntos extremos (3).

\section{RECOMENDACIONES}

1. Realizar estudios moleculares de purificación que permitan encontrar un antígeno específico a $M$. leprae.

2. Cuando se use el ASML hacerlo paralelo a PPD, que permita la comparación bien controlada de las respuestas específicas como no específicas.

3. También sumado a lo anterior o en forma independiente realizar estudios con marcadores genéticos que permiten dilucidar asociaciones genéticas con el grado de resistencia o susceptibilidad.

\section{SUMMARY}

Cross reactions to purified protein derivative, PPD, of tuberculin and Mycobacterium leprae soluble antigen, MLSA, were measured among children of 6-14 years old from two areas. In the first zone, Pascua (Easter) Island, leprosy is endemic while in the second one, Santiago de Chile on the continent, leprosy is non endemic. Children in each locality were simultaneously inoculated with PPD and MLSA on right and left forearms, respectively. Readings were performed $48 \mathrm{~h}$ later. Statistic analyses demonstrated and evident cross reaction between the antigens. A significant greater reaction was also observed in endemic area (Pascua Island) than in non endemic area (Santiago) both for PPD and MLSA.

Keywords: PPD, ASML and correlation.

\section{BIBLIOGRAFIA}

1. Leprosy Review. Special issue, British Leprosy Relief Association. June, 1983.

2. Rodríguez GL. Estudio inmunológico de la población de Isla de Pascua para detectar infección hanseniásica. Rev. Med Chile, 1985; 113: 183-185.

3. Seminario Bolivariano sobre control de la lepra. Investigación científica y control de la lepra. Caracas, 12-14 septiembre, 1983.

4. Ministerio de Salud Pública Chile. Informes de lepra en Isla de Pascua, 1959, 1969, 1978, junio-diciembre, 1983.

5. Rose NR. Manual of clinical laboratory inmunology. Tercera edición, American Society of Microbiology, 1986. 